\title{
A Review of Employees Collective Turnover Research and Prospects
}

\author{
Yu-Huan $\mathrm{Bi}^{1, a}$ \\ ${ }^{1}$ Business School,East China University of Science \& Technology, \\ Shanghai 200237 China \\ a josie bi1016@163.com
}

Key words: employees collective turnover; concept; occurrence mechanism; outcome

\begin{abstract}
In recent years, employees collective turnover has occurred frequently, which affected the stable operation and development of enterprises seriously. Collective turnover is different from individual turnover, but traditional theory of turnover mainly focus on the individual level, and what we know about collective turnover is not enough. This paper reviewed the previous related studies and then concluded the concept, occurrence mechanism and outcomes of employee collective turnover respectively. Further, some future direction were prospected.
\end{abstract}

\section{Introduction}

Employee turnover is one of the most widely studied phenomena in the field of human resource management and organizational behavior. However, in recent years, a new phenomenon of turnover---collective turnover frequently appeared, and gradually attracted attention. For example, July 2014, Jahwa occurred employees collective turnover(including general manager), this phenomenon seriously affects the development of the company[1]. Collective turnover's occurrence mechanism and effect are different from individual turnover. In view of the difference between collective and individual turnover, it is not easy to apply the individual turnover theory to explain the phenomenon of collective turnover[2].Therefore, the problem of collective turnover has become a new problem to be solved.

\section{Definition of collective turnover}

For the collective turnover, there is no unified definition. The existing research mainly defines and describes it from two aspects:the process perspective and the result perspective.

Process perspective definition focuses on collective turnover occurrence mechanism, according to whether the emphasis on turnover interaction between individuals can be classified into two categories: one that the collective turnover is a plurality of individual due to certain reasons common away, without taking into account the turnover between the individual existence of mutual influence. For example, $\mathrm{He}$ and his colleagues defined collective turnover as a group of organizational members with similar dissatisfaction, ideas and goals to leave the organization together with the common values and goals[3]; Another type of special emphasis on the interaction and contact between individuals. For example, Gmysbery and Abrahams believe that when the higher the closer the relationship and interaction between members, his leader is more personality in the informal organization,[4]. And Bartunek et al also believe that the collective turnover is two and more members of the organization based on the sharing of social processes and decision-making, in a similar time to leave an organization[5].

Result perspective emphasizes collective turnover lead to the organization of the human capital loss, there are mainly two kinds of views: a only to emphasize the "quantity" of organizational human capital in terms of loss, the collective turnover simply equal to employee turnover rate[6]; 
another aspect of human capital "quality" is also included in the scope of consideration, because the mere turnover rate of different individuals to ignore the difference between the individual and the organization of the importance of [2]. Based on this, Nyberg and Ployhart believe that collective turnover refers to the organization of employee knowledge, skill, ability etc. (KSAs) in terms of quality and quantity of two aspects of the loss. Among them, the number of employee turnover rate, quality refers to the loss of KSAs types and levels of staff[2].

\section{The occurrence mechanism of collective turnover}

Different process models are proposed for the occurrence mechanism of collective turnover. Among them, the representative of the model is the turnover contagion model and the process model of collective turnover.

\section{The turnover contagion model}

The turnover contagion model is proposed by Felp et al, which is considered to have a "snowball effect", which means that an employee's departure will trigger other employees to leave through the social contagion process[7]. Its theoretical basis is the theory of social comparison, that in reality, people's understanding and evaluation of its characteristics (such as ability, intelligence, etc.) more often than not based on objective criteria, but by compared with other people around, which is a widespread phenomenon of social psychology [8].

\section{The process model of collective turnover.}

The process model of collective turnover was first proposed by Bartunek et al[5], then the model was modified by Dong and Wang[9]. Bartunek et al believes that collective turnover is an external solution to the group's negative emotions, including the voice and exit of two links. In the "voice" link, the organization of a certain / some factors trigger a certain / some of the employee's discontent, then the employee complaints or suggestions to the superior; If they are satisfied with the solution, then the discontent disappeared, or they will enter the "exit" link. In "exit" link, not satisfied with the individual by group sensemaking, emotional contagion channels in the population spread dissatisfaction with their, make personal emotion into groups with common negative emotions. Feel the improvement of the hopeless members, and they will make a decision to leave; some members of the turnover in turn may shake the remaining members to stay on the determination, triggering wider turnover.

The modified model of collective turnover can occur through two paths: one is the path of "push". In a group of one or more members of the "push" effect of negative emotion, and then choose a different way. One is positive feedback to the superior. if no satisfactory answer, their negative experiences through emotional contagion and group sensemaking to the collective conduction and form shared negative perception and emotions; The other is a negative response, not satisfied with the individual directly complaints directly to the group about their own dissatisfaction, and then the formation of a total of negative feelings and emotions in the group. If the employee's negative perception of the organization and continue to strengthen the emotional, leading to employee to enter the exit link, and then produce collective turnover. Two is the path of "pull". One or many of the members by the "pull" effect positive turnover of emotion and cognition, through sharing and dissemination of collective and raised the prospect of collective positive cognition and emotion to quit, after staff cognitive and emotional overlay and enhancement will enter the "exit" link, collective turnover will appear. "Pull" factors caused by the collective turnover may again will by "push" factors in the development process. 


\section{The result of collective turnover}

Existing research mainly discusses the collective turnover and three outcome variables: first, the performance of the company;second, production efficiency; third, and customer related results. Most studies suggest that the collective turnover of the linear relationship between the variables and the results, there are some paper or confirmed the nonlinear relation between them.

\section{The linear relationship between collective turnover and outcome variables}

According to the related research, the linear relationship between the collective turnover and the outcome variables is mainly negative correlation. Because the collective turnover means that the organization of human capital loss and normal operations are disrupted, recruitment, selection and increased social costs of new employees, and then will have a negative impact on the organization [10]. Many empirical studies support this view. For example, Ton and Huckman found that collective turnover will bring about a decline in organizational profit margins[11]. Shaw et al found that the turnover rate of each unit will lead to a decline in sales of about $\$ 1500$ per unit[12]. And research has confirmed that high turnover rate and longer waiting time for customers, lower customer satisfaction related [13].

\section{The nonlinear relationship between collective turnover and outcome variables}

There are two kinds of nonlinear relationships between collective turnover and outcome variables: One is the decline of negative correlation, Second is the inverted U type.

For "decreasing negative correlation",some researchers gives the following explanation: the human capital stock which is needed to maintain the normal organization performance as the critical value. When the turnover rate is low, remaining in the tissue of human capital stock at the critical value, normal performance still can be maintained. Leaving the loss of human capital makes normal performance on the extra performance loss; and when the turnover rate is higher, organizational human capital stock has been depleted, performance has been reduced to a low level, the additional staff turnover is unlikely to result in a significant decline in performance and curve tends to be gentle. Moreover, when the turnover rate is high, the organization will usually take certain measures to remedy, such as frequent recruitment and so on, in order to weaken its negative impact [12].

For "inverted U relationship", the relevant research believes that when the employee turnover rate is low, due to the lack of new employees, new ideas into the organization is more conservative and closed, and thus lead to lower performance [14]; when the employees turnover rate from low rise gradually to the appropriate level of time, gradually increase the organization innovation, flexibility and adaptability, performance will gradually improve ; when the employee turnover rate is more than the appropriate level and higher, the negative effects will exceed the positive effects and organizational performance with the loss rate of excessive decreases.

\section{Summary and Prospect}

\section{Constructing a special collective turnover theory}

Previous studies have pointed out that the collective turnover is different from the individual separation, so we can not simply apply the theory of individual turnover to explain. However, many research on collective turnover is still based on the theory of individual turnover. Therefore, although the individual turnover research results have some reference value for collective turnover, but can not completely copy or mechanically apply, to combine the characteristics of collective turnover for specific analysis. This needs to be based on the relevant theory from the group or organizational level to build a special the theory of collective turnover ,such as Nyberg and Ployhart[2] in cross level theory and human capital theory based on the construction of the "context 
emergent turnover theory", explain the difference between individual and collective turnover, and the collective turnover path that affect the organization performance. Future research needs to continue its efforts in this regard.

\section{Explore the positive impact of collective turnover}

Current related research is mainly concerned and confirmed the negative impact of collective turnover, but it may also have a positive impact: On the one hand, the research on the relationship between turnover and organizational performance of inverted $U$ show that the appropriate level of collective turnover is beneficial to the organization. But the hypothesis has not been supported by sufficient empirical support; On the other hand, the current study on the effect of collective turnover only considers the effect of collective turnover on turnover organization, without consideration of the effect of collective turnover on turnover individual, as well as industry or social development and so on. Therefor,the future research needs to take into account the negative impact of collective turnover and positiveimpact, in the suppression of its negative effects while using its positive role, in order to achieve a balance between the two points.

\section{References}

[1]Information on http://finance.sina.com.cn/stock/s/20140718/192919752574.shtml

[2]Nyberg A J,Ployhart R E. Context-emergent Turnover(CET) Theory: A Theory of collective turnover[J]. Academy of Management Review, 2013, 38(1): 109-131.

[3]He X G, Han J, Shen Y. The present situation and motivation of enterprise collective turnover in transition period $[\mathrm{J}]$. The development of human resources in China, 2006, (6):58-63

[4]Groysbeerg R B,Abrahams R. Lift outs:How to Acquire a High_functioning Team [J]. Harvard Business Review,2006,84(12):133-145.

[5]Bartunek J M, Huang Z, Walsh I J. The development of a process model of collective turnover[J]. Human Relations, 2008, 61(1): 5-38.

[6]Gerorge J M, Bettenhaysen K. Understanding prosocial behavior, sales performance, and turnover: a group-level analysis in a service context[J]. Journal of applied psychology, 1990, 75(6): 698-709.

[7]Felp W, Mitchell T R, Hekman D R et al.Turnover contagion: How coworkers' job embeddedness and job search behaviors influence quitting[J]. Academy of Management Journal,2009,52(3): 545-561

[8]Festunger LA. A theory of social comparison process[J]. Human Relations. 1954,7(2): 117-140

[9]Dong B W, Wang S.Research on the model of collective turnover process [A] .Fourth (2014) China management academic year, organizational behavior and Human Resource Management Branch.[C] ,2014.

[10]Dess G G,Shaw J D. Voluntary turnover, social capital, and organizational performance[J]. Academy of Management Review, 2001,26(3): 446-456

[11]Ton Z,Huckman R S. Managing the impact of employee turnover on performance: The role of process conformance[J]. Organization Science,2008,19(1): 56-68.

[12]Shaw J D,Gupan N,Delery J E. Alternative conceptualizations of the relationship between voluntary turnover and organizational performance[J].Academy of Management Journal,2005,48(1): 50-68.

[13]Hurley R F,Estelami H. An exploratory study of employee turnover indicators as predictors of customer satisfaction[J]. Journal of Services Marketing, 2007,21(3): 186-199.

[14]Dalton D R,Todor W D. Turnover turned over: An expanded and positive perspective[J]. Academy of Management Review,1979, 4(2): 225-235. 